\title{
Edentulismo afeta hemodinâmica mandibular em indivíduos idosos: estudo in vivo
}

\section{Edentulism affects mandibular hemodynamics in elderly}

\author{
Marina GazZANo BALAdI \\ Doutora em Odontologia pela Faculdade de \\ Odontologia da Universidade de São Paulo
}

EMIKo SAITO ARITA

Profa. Doutora do Departamento de Estomatologia da Faculdade de Odontologia da Universidade de São Paulo

EduARdo Massaharu AoKI Mestre em Odontologia pela Faculdade de Odontologia da Universidade de São Paulo

Arthur Rodrigues Gonzalez Cortes Doutor em Odontologia pela Faculdade de Odontologia da Universidade de São Paulo

Daniela Miranda Richarte de Andrade Salgado Mestre em Diagnóstico Bucal pela Faculdade de Odontologia da Universidade da Paulista

Claudio Costa

Prof. Doutor do Departamento de Estomatologia da Faculdade de Odontologia da Universidade de São Paulo

Claudio Fróes de Freitas

Prof. Doutor do Departamento de Estomatologia da Faculdade de Odontologia da Universidade de São Paulo

\begin{abstract}
RESUMO
Introdução: A ultrassonografia apresenta um papel importante na caracterização da hemodinâmica mandibular e fornece a possibilidade de análise de alterações na vascularização relacionadas ao edentulismo.

Objetivo: Analisar a hemodinâmica de artérias mandibulares responsáveis pela nutrição do tecido ósseo por meio da Ultrassonografia modo B e Doppler em pacientes idosos dentados e edêntulos. Material e Método: 60 exames de ultrassonografia das artérias Alveolar Inferior (AAI) e Mentual (AM) foram avaliados em dois grupos de pacientes: $\mathrm{G} 1-15$ pacientes idosos edêntulos e $\mathrm{G} 2-15$ pacientes dentados, ambos com idade média cronológica acima de 60 anos. As variáveis analisadas foram: Índice de Resistência Vascular (IRV), Índice de Diâmetro (ID), Velocidade de Pico Sistólico (VPS) e Hemodinâmica. O teste estatístico utilizado foi o Teste t não pareado a um nível de significância de $5 \%$. Resultados: Foi demonstrado que os valores da hemodinâmica de AAl e AM, lados esquerdo e direito, foram menores no G1 do que no G2. Nos pacientes edêntulos com atrofia mandibular, o fluxo sanguíneo da AM foi menor quando comparado com o da AAI. Conclusão: Nos pacientes edêntulos, a atrofia mandibular modifica o fluxo sanguíneo nas artérias mandibulares.
\end{abstract}

Palavras-chave: Ultrassonografia; Hemodinâmica; Efeito Doppler; Mandíbula. 


\begin{abstract}
Introduction: The ultrasonography has an important role in the characterization of hemodynamics and provides the possibility to analyze changes in vascularization. Objective: To analyze the hemodynamic in mandibular arteries responsible for the nutrition of bone tissue through ultrasound $B$ mode and Doppler. Material and Methods: The results of 60 ultrasound exams of the Arteries Inferior Alveolar (AIA) and Mentual (MA) were evaluated in two groups of patients: G1 - 15 edentulous elderly patients and G2 - 15 patients both with average chronological age above 60 years old. The variables analyzed were: Vascular Resistance Index (VRI), Diameter Index (DI), Peak Systolic Velocity (PSV) and Hemodynamics. The statistical test used was the $T$ test unpaired at $5 \%$ significance level. Results: It has been shown that the hemodynamic values of AIA and MA, left and right sides were lower in G1 compared to G2. In edentulous patients with mandibular atrophy, blood flow of MA was lower when compared to that of AIA. Conclusion: In edentulous patients, the mandibular atrophy modifies the blood flow in mandibular arteries.
\end{abstract}

Key-words: Ultrasonography; hemodynamics; Doppler Effect; Mandible.

\section{INTRODUÇÃO}

O edentulismo em pacientes idosos gera a diminuição do suprimento sanguíneo em mandíbulas atróficas. ${ }^{1}$ Pesquisadores, ${ }^{2}$ ao investigarem a hemodinâmica mandibular por meio da ultrassonografia modo B e Doppler, relataram que a diminuição do lúmen da $\mathrm{AAl}$ está relacionada com a redução do suprimento sanguíneo e, por conseguinte, com a atrofia mandibular.

Nesse contexto, a ultrassonografia modo B e Doppler tem um importante papel na caracterização da hemodinâmica sanguínea, ${ }^{3}$ permitindo a observação do fluxo dos vasos sanguíneos mandibulares, responsáveis pela nutrição, além de direcionar o diagnóstico clínico e os procedimentos cirúrgicos. ${ }^{4}$

Esse exame é um método de diagnóstico por imagem, rápido, versátil, de utilização relativamente simples, de custo acessível e não invasivo. ${ }^{5}$ Apresenta, pelo modo $B$, a anatomia em imagens seccionais, que podem ser adquiridas em qualquer orientação espacial; não causa efeitos ionizantes e enriquece o diagnóstico, possibilitando o estudo da hemodinâmica corporal, por meio do efeito Doppler, que analisa o volume sanguíneo e a arquitetura vascular de vasos importantes em diversos órgãos vitais, em tempo real. ${ }^{6}$

Atualmente, os procedimentos de reabilitação oral por meio de implantes dentários, nos pacientes idosos edêntulos, geram uma preocupação com a vascularização mandibular, quantidade e qualidade óssea, visando o sucesso do tratamento. Sendo assim, o objetivo deste estudo foi o de caracterizar a hemodinâmica mandibular em pacientes idosos dentados e edêntulos, por intermédio da ultrassonografia modo B e Doppler.

\section{Materiais e Métodos}

Este estudo exploratório foi iniciado após o parecer de aprovação do Comitê de Ética (Número do Parecer: 166.119; CAAE 08532012.8.0000.0075) em 30/11/2012.

\section{Seleção da Amostra}

Os pacientes que participaram da presente pesquisa faziam parte do Projeto Envelhecer Sorrindo, da Faculdade de Odontologia da Universidade de São Paulo - FOUSP. Trinta pacientes participaram do estudo, após assinarem o Termo de Consentimento Livre e Esclarecido, e nenhum paciente se recusou a participar do estudo.

Os pacientes foram divididos em dois grupos: G1 - 15 pacientes idosos edêntulos totais na mandíbula com atrofia de rebordo ósseo alveolar observada por meio de uma radiografia panorâmica recentemente adquirida. G2 - 15 pacientes adultos idosos dentados com a presença obrigatória dos primeiros molares em ambos os lados. 
Critérios de Inclusão: pacientes com idade acima de 60 anos, saudáveis, selecionados por meio de dados clínicos coletados por intermédio do exame clínico (anamnese).

Critério de Exclusão: indivíduos portadores de alterações sistêmicas (varizes, hipotireoidismo, fibromialgia, síndrome de Reynaud, doenças reumáticas, respiratórias, cardiovasculares que modificassem o estado geral das artérias.

\section{Ultrassonografia}

Foi realizada a ultrassonografia intraoral em ambos os lados das artérias: Alveolar Inferior (AAI) no forame mandibular e Mentual (AM) no forame mentual, totalizando 60 exames de ultrassonografia modo B e Doppler, tendo sito observadas quatro variáveis quantitativas:

- Índice de Resistência Vascular (IRV)

- Índice do Diâmetro (ID) em milímetros (mm)

- Velocidade de Pico Sistólico (VPS) em centímetros por segundos $(\mathrm{cm} / \mathrm{s})$

- Hemodinâmica (fluxo de sangue da artéria) foi calculada a partir da seguinte fórmula:

$$
\text { Hemodinânica }=\frac{(I D / 10)^{2}+\pi \times \text { VPS }}{4}\left(\mathrm{~cm}^{3} / \mathrm{s}\right)
$$

As quatro variáveis supracitadas foram comparadas segundo os lados (direito e esquerdo), grupos (edêntulos e dentados) e artérias (AAl e $A M)$ e submetidas a análises estatísticas.

\section{Equipamento}

As imagens foram obtidas por um aparelho de ultrassom portátil Terason $\mathrm{t} 3000$ (Terason, divisão da Teratech Corporation, EUA), utilizando um transdutor endocavitário, modelo 8EC4, com frequência variável de $4-8 \mathrm{MHz}$. Intraoralmente foi utilizado o invólucro de látex (Maditex® - Maditex Ind. Com. de Latex Ltda.), com gel para a proteção do transdutor.

Os exames intraorais foram realizados por um operador médico radiologista, com pelo menos 20 anos de experiência em ultrassonografia. Os pacientes edêntulos e dentados foram posicionados no equipo odontológico com inclinação de $45^{\circ} \mathrm{em}$ relação ao solo. Iniciou-se a investigação ultrassonográfica com o transdutor posicionado na região do forame mandibular e mentual, caracterizando as variáveis VRI, VPS e ID.

$A$ imagem a seguir foi produzida e avaliada em tempo real pela ultrassonografia modo $B$ e Doppler (Figura 1).

Figura 1 - Imagem produzida pelo ultrassom, mostrando a captura do sinal Doppler da artéria mentual direita.

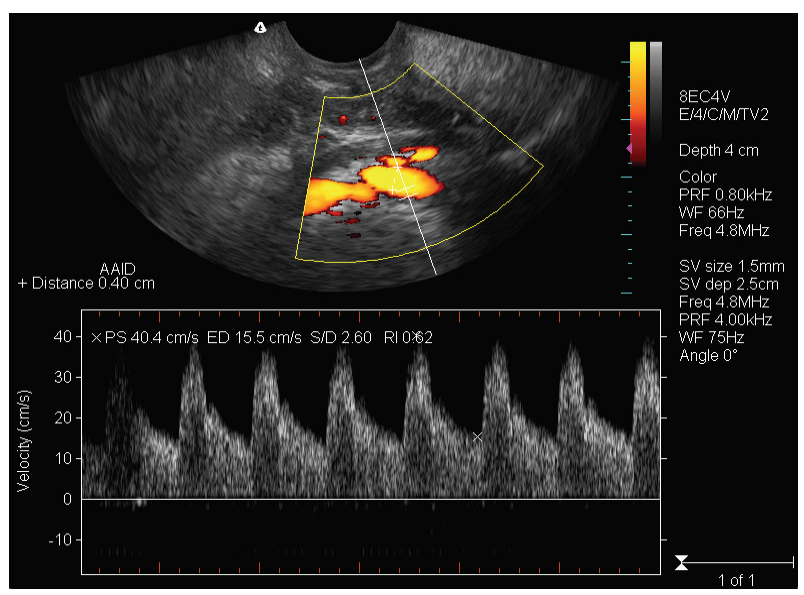

\section{Análise Estatística}

Os resultados obtidos foram tabulados no software Excel (Microsoft Corp., EUA, 2011) e as análises estatísticas foram realizadas com o auxílio do software BioEstat 5.0 (Universidade Federal do Amazonas, Instituto Mamirauá $)^{7}$ por meio da análise de Teste $t$ não pareado, onde os valores com $\mathrm{p}<005$ foram considerados para indicar significância estatística.

\section{Resultados}

Trinta pacientes foram incluídos no estudo. A média de idade dos pacientes foi: $\mathrm{G} 1=$ $7133 \pm 764$ e G2 = 6546 \pm 794 .

A análise estatística mostrou que, ao se comparar os dois grupos, G1 e G2, todos 
os parâmetros estudados apresentaram diferenças significantes na análise da AM. Esses parâmetros foram: IRV $(p=00003)$; ID $(p<00001)$; VPS $(p=00004)$ e Hemodinâmica $(p<00001)$. Entretanto, na análise da AAI, os resultados não mostraram diferenças estatisticamente significantes $(p>005)$ na comparação dos grupos estudados (Tabela 1).

Quando comparados os lados direito e esquerdo, não houve diferenças significantes ( $p>005)$, mostrando que essa variável (lado) não interfere na análise do fluxo sanguíneo da AAl e AM.

\section{Dıscussão}

Edentulismo e atrofia mandibular são importantes sinais clínicos em pacientes idosos, sua prevenção e conduta clínica é de fácil controle para o cirurgião-dentista (CD), devido ao reduzido suprimento sanguíneo mandibular originado por fonte interna (AAI) e externa (periósteo), responsáveis pela prevenção da atrofia da crista mandibular. 1,8,9,10 $^{2}$

Os resultados foram estatisticamente significantes $(p<00001)$, sugerindo que pacientes idosos edêntulos, com atrofia mandibular, apresentam fluxo sanguíneo reduzido, demonstrado pela ultrassonografia modo B e Doppler, que forneceu parâmetros hemodinâmicos da região estudada, com potencial importância diagnóstica, o que está de acordo com os estudos prévios. ${ }^{1,11}$

Assim como no presente estudo, outros autores ressaltaram também a diminuição do fluxo da artéria mentual em indivíduos idosos edêntulos, a qual estaria associada à atrofia óssea alveolar, uma vez que ela é uma continuação da AAI..$^{1,10}$

Diferentemente destes resultados, algumas pesquisas realizadas com a interrupção do fluxo da AAI propõem que ocorreria um aumento do desenvolvimento vascular da artéria mentual e sublingual, passando a constituir as fontes principais de nutrição para o corpo mandibular, ${ }^{11}$ o que confirma a importância do estudo da AM. Outros autores acrescentaram que esse processo de nutrição mandibular se daria pelo aumento do diâmetro da artéria mentual e por meio das anastomoses colaterais com fluxo reverso, a partir da artéria submentual, ramo da artéria facial. ${ }^{12}$

Estudos realizados por meio da ultrassonografia modo $B$ e Doppler que

Tabela 1 - Análise das variáveis das artérias (AAI e AM) quanto aos grupos de pacientes (edêntulos e dentados).

\begin{tabular}{|l|l|l|l}
\hline \multicolumn{1}{c|}{ AAI } & \multicolumn{1}{c|}{ DENTADO } & \multicolumn{1}{c|}{ EDÊNTULO } & \multicolumn{1}{c}{ P VALOR } \\
\hline IRV & $078(006)$ & $081(005)$ & 010 \\
\hline ID & $207(038)$ & $206(036)$ & 047 \\
\hline VPS & $2889(1077)$ & $2595(850)$ & 020 \\
\hline HEMODINÂMICA & $111(074)$ & $084(038)$ & 017 \\
\hline
\end{tabular}

\begin{tabular}{|l|l|l|l}
\hline \multicolumn{1}{|c|}{ AM } & \multicolumn{1}{c|}{ DENTADO } & \multicolumn{1}{c}{ EDÊNTULO } & \multicolumn{1}{c}{ P VALOR } \\
\hline IRV & $067(018)$ & $027(033)$ & $00003^{*}$ \\
\hline ID & $110(0.31)$ & $041(0.51)$ & $<00001^{*}$ \\
\hline
\end{tabular}

*Teste T - Significância a 005. 
avaliem a vascularização tecidual mandibular utilizando como parâmetros o fluxo sanguíneo nos pacientes idosos edêntulos, para posterior reabilitação oral mandibular por implante e próteses dentárias, são de grande importância. ${ }^{13,14}$ Esse método de aquisição de imagem agrega valores importantes, como a possibilidade de detecção de padrões do fluxo sanguíneo normal (periférico) e da existência de fluxo anormal devido a possíveis lesões, facilitando ao médico e ao cirurgião-dentista, em tempo real, informações por imagens em alta definição obtidas por multifrequência por meio de sondas ou transdutores que operam a uma frequência de $8-11 \mathrm{MHz}$, contribuindo para o diagnóstico da vascularização. $4,11,15,16,17$ Sendo assim, a ultrassonografia é uma importante ferramenta de diagnóstico de custo acessível, indolor, pouco invasiva, podendo ser repetida com frequência para o monitoramento das intervenções terapêuticas. É inócua, fornecendo imagens dos tecidos moles e da arquitetura vascular em tempo real e tem sido muito utilizada atualmente para a exploração dos tecidos orais e hemodinâmica dos grandes vasos da cabeça e pescoço. ${ }^{18}$

Com base nos resultados quantitativos deste estudo e nos achados característicos, sugerese que o edentulismo produza alterações vasculares que poderiam estar associadas à consequente atrofia óssea mandibular.

\section{Conclusão}

O fluxo sanguíneo da AM foi menor nos pacientes edêntulos do que nos pacientes dentados diferentemente da AAI que não apresentou alterações significantes neste estudo entre os dois grupos.

\section{Agradecimentos}

Agradecemos à Prof. ${ }^{a}$ Dr. $^{\text {a }}$ Marlene Fenyo Soeiro de Matos Pereira, pelo seu encorajamento gentil e apoio e Prof. ${ }^{a}$ Dr. ${ }^{a}$ Maria
Luiza Moreira Arantes Frigério, coordenadora do Projeto Envelhecer Sorrindo, da Faculdade de Odontologia da Universidade de São Paulo - FOUSP, pela disponibilidade da amostra.

\section{REFERÊNCIAS}

1. Eisemann B., Johnson LR, Coll JR. Ultrasound measurement of mandibular arterial blood supply. Techniques for defining ischemia in the pathogenesis of alveolar ridge atrophy and tooth loss in the elderly. J Oral Maxillofac Surg., 2005; 63: 28-35.

2. Bartczyszyn M., Wieckiewixz W., Wieckiewixz M. Blood flow in mandibular vessels - its evaluation and prognostic implications including Doppler techniques - review of literature. Czas Stomatol., 2010; 63(5): 320-6.

3. Baladi MG, Tucunduva MJAPS, Tucunduva Neto R., Cortes ARG, Aoki EM, Arita ES, Freitas CF. Effectiveness of ultrasonography in detecting intraosseous vascularization: an in-vitro study. Clinical and Laboratorial Research in Dentistry, 2014; 20: 160-65.

4. Oeppen RS, Gibson D., Brennan PA. An update on the use of ultrasound imaging in oral and maxillofacial surgery. British Jounal of Oral and Maxillofacial Surgery, 2010; 48: 412-18.

5. Jones JK, Frost DE. Ultrasound as a diagnostic aid in maxillofacial surgery. Oral Surg. Oral Med. Oral Pathol. Oral Radiol. Endod., 1984; 57(6): 589-94.

6.Schmidt G. Guia de ultrassonografiadiagnóstico por imagem. Trad. Müller R., Porto Alegre; Artmed; 2010.

7. Ayres M., Ayres Jr. M., Ayres DL, Santos AS. 2007, Bioestat 5.0: Aplicações estatísticas nas áreas da ciências biológicas e médicas. Brasília, CNPq/Conservation International, Manual do programa Bioestat.

8. Castelli W. Vascular architecture of the human adult mandible. J. Dent. Res. 1963; 42: 786-92.

9. Chanavaz M. Anatomy and histophysiology of the periosteum: quantification of the periosteal blood supply to the adjacent bone with $85 \mathrm{Sr}$ and gamma spectrometry. J Oral Implantol., 1995; 21: 214-19. 
10. Baladi MG, Neto RRCMT, Cortes ARG, Aoki EM, Arita ES, Freitas CF. Ultrasound analysis of mental artery flow in elderly patients: a casecontrol study. Dentomaxillofac Radiology, 2015; 44: 1-5.

11. Ethunandan M., Birch AA, Evans BT, Goddard JR. Doppler sonography for the assessment of central mandibular blood flow. Br. J. Oral. Maxillofac Surg., 2000; 38: 294-8.

12. Castelli WA, Nasjlet CE, Díaz-Pérez R. Interruption of the arterial inferior alveolar flow and its effects on mandibular collateral circulation and dental tissues. J. Dent. Res., 1975; 54: 708-15.

13. Güler AU, Sumer M., Sumer P., Biçer I. The evaluation of vertical heights of maxillary and mandibular bones and the locations of anatomic landmarks in panoramic radiographs of edentulous patients for implant dentistry. J. Oral Rehabil., 2005; 32(10): 741-6.

14. Panchbhai AS. Quantitative estimation of vertical heights of maxillary and mandibular jawbones in elderly dentate and edentulous subjects. Spec. Care Dentist., 2013; 33(2): 62-9.

15. Sniezek JC. Head and Neck Ultrasound: Wy Now? Otolaryngol. Clin. N. Am., 2010; 43: 1143-47.

16. Mahamoud AM, Ngan P., Crout R., Mukdadi OM. High-resolution 3D ultrasound jawbone surface imaging for diagnosis of periodontal bony defects: an in vitro study. Annals of Biomedical Engeneering, 2010; 38(11): 3409-22.

17. Yuasa K., Kawuzu T., Nagata T., Kanda S., Ohishi M., Shirasuna K. Computed tomography and ultrasonography of metastatic cervical lymph nodes in oral squamous cell carcinoma. Dentomaxillofac Radiol., 2000; 29: 238-44.

18. Salmon B., Le Denmat D. Intraoral ultrasonography: development of a specific high-frequency probe and clinical pilot study. Clin. Oral Investig., 2012; 16(2): 643:9.

Submetido em: 31-3-2016

Aceito em: 8-6-2016 